\title{
Introduction to Turing Machines
}

\author{
Jing-Chao Chen \\ Bell Labs Research China \\ Lucent Technologies \\ Bejing
}

\author{
Yatsuka Nakamura \\ Shinshu University \\ Nagano
}

Summary. A Turing machine can be viewed as a simple kind of computer, whose operations are constrainted to reading and writing symbols on a tape, or moving along the tape to the left or right. In theory, one has proven that the computability of Turing machines is equivalent to recursive functions. This article defines and verifies the Turing machines of summation and three primitive functions which are successor, zero and project functions. It is difficult to compute sophisticated functions by simple Turing machines. Therefore, we define the combination of two Turing machines.

MML Identifier: TURING_1.

WWW: http://mizar.org/JFM/Vol13/turing_1.html

The articles [18], [9], [24], [2], [21], [3], [15], [1], [22], [14], [19], [17], [6], [7], [12], [4], [11], [20], [10], [8], [16], [23], [13], [25], and [5] provide the notation and terminology for this paper.

\section{PRELIMINARIES}

In this paper $n, i, j, k$ are natural numbers.

Let $A, B$ be non empty sets, let $f$ be a function from $A$ into $B$, and let $g$ be a partial function from $A$ to $B$. Then $f+\cdot g$ is a function from $A$ into $B$.

Let $X, Y$ be non empty sets, let $a$ be an element of $X$, and let $b$ be an element of $Y$. Then $a \longmapsto b$ is a partial function from $X$ to $Y$.

Let $n$ be a natural number. The functor $\operatorname{Seg}_{M} n$ yielding a subset of $\mathbb{N}$ is defined as follows:

(Def. 1) $\operatorname{Seg}_{M} n=\{k: k \leq n\}$.

Let $n$ be a natural number. One can verify that $\operatorname{Seg}_{M} n$ is finite and non empty.

One can prove the following propositions:

(1) $k \in \operatorname{Seg}_{M} n$ iff $k \leq n$.

(2) For every function $f$ and for all sets $x, y, z, u, v$ such that $u \neq x$ holds $(f+\cdot(\langle x, y\rangle \longmapsto z))(\langle u$, $v\rangle)=f(\langle u, v\rangle)$.

(3) For every function $f$ and for all sets $x, y, z, u, v$ such that $v \neq y$ holds $(f+\cdot(\langle x, y\rangle \longmapsto z))(\langle u$, $v\rangle)=f(\langle u, v\rangle)$.

In the sequel $i_{1}, i_{2}, i_{3}, i_{4}$ are elements of $\mathbb{Z}$.

One can prove the following propositions:

(4) $\sum\left\langle i_{1}, i_{2}\right\rangle=i_{1}+i_{2}$. 
(5) $\sum\left\langle i_{1}, i_{2}, i_{3}\right\rangle=i_{1}+i_{2}+i_{3}$.

(6) $\sum\left\langle i_{1}, i_{2}, i_{3}, i_{4}\right\rangle=i_{1}+i_{2}+i_{3}+i_{4}$.

Let $f$ be a finite sequence of elements of $\mathbb{N}$ and let $i$ be a natural number. The functor $\operatorname{Prefix}(f, i)$ yielding a finite sequence of elements of $\mathbb{Z}$ is defined as follows:

(Def. 2) $\operatorname{Prefix}(f, i)=f\lceil\operatorname{Seg} i$.

The following propositions are true:

(7) For all natural numbers $x_{1}, x_{2}$ holds $\sum \operatorname{Prefix}\left(\left\langle x_{1}, x_{2}\right\rangle, 1\right)=x_{1}$ and $\sum \operatorname{Prefix}\left(\left\langle x_{1}, x_{2}\right\rangle, 2\right)=$ $x_{1}+x_{2}$.

(8) For all natural numbers $x_{1}, x_{2}, x_{3}$ holds $\sum \operatorname{Prefix}\left(\left\langle x_{1}, x_{2}, x_{3}\right\rangle, 1\right)=x_{1}$ and $\sum \operatorname{Prefix}\left(\left\langle x_{1}, x_{2}\right.\right.$, $\left.\left.x_{3}\right\rangle, 2\right)=x_{1}+x_{2}$ and $\sum \operatorname{Prefix}\left(\left\langle x_{1}, x_{2}, x_{3}\right\rangle, 3\right)=x_{1}+x_{2}+x_{3}$.

\section{Definitions And Terminology For Turing Machine}

We consider Turing machine structures as systems

$\langle$ symbols, control states, a transition, an initial state, an accepting state $\rangle$,

where the symbols and the control states constitute finite non empty sets, the transition is a function from [: the control states, the symbols:] into [: the control states, the symbols, $\{-1,0,1\}:]$, and the initial state and the accepting state are elements of the control states.

Let $T$ be a Turing machine structure. A state of $T$ is an element of the control states of $T$. A tape of $T$ is an element of (the symbols of $T)^{\mathbb{Z}}$. A symbol of $T$ is an element of the symbols of $T$.

Let $T$ be a Turing machine structure, let $t$ be a tape of $T$, let $h$ be an integer, and let $s$ be a symbol of $T$. The functor Tape-Chg $(t, h, s)$ yields a tape of $T$ and is defined by:

(Def. 3) Tape-Chg $(t, h, s)=t+\cdot(h \longmapsto s)$.

Let $T$ be a Turing machine structure. A State of $T$ is an element of [: the control states of $T$, $\mathbb{Z}$, (the symbols of $T)^{\mathbb{Z}}:$. A transition-source of $T$ is an element of [: the control states of $T$, the symbols of $T$ :]. A transition-target of $T$ is an element of [: the control states of $T$, the symbols of $T$, $\{-1,0,1\}:]$.

Let $T$ be a Turing machine structure and let $g$ be a transition-target of $T$. The functor offset $(g)$ yields an integer and is defined as follows:

(Def. 4) $\operatorname{offset}(g)=g_{3}$.

Let $T$ be a Turing machine structure and let $s$ be a State of $T$. The functor Head(s) yields an integer and is defined as follows:

(Def. 5) $\operatorname{Head}(s)=s_{2}$.

Let $T$ be a Turing machine structure and let $s$ be a State of $T$. The functor $s$-target yielding a transition-target of $T$ is defined by:

(Def. 6) $\quad s$-target $=($ the transition of $T)\left(\left\langle s_{\mathbf{1}},\left(s_{\mathbf{3}}\right.\right.\right.$ qua tape of $\left.\left.\left.T\right)(\operatorname{Head}(s))\right\rangle\right)$.

Let $T$ be a Turing machine structure and let $s$ be a State of $T$. The functor Following $(s)$ yields a State of $T$ and is defined as follows:

(Def. 7) $\quad$ Following $(s)=\left\{\begin{array}{l}\left\langle s-\operatorname{target} \mathbf{1}, \operatorname{Head}(s)+\operatorname{offset}(s-\operatorname{target}), \operatorname{Tape}-\operatorname{Chg}\left(s_{\mathbf{3}}, \operatorname{Head}(s), s-\operatorname{target}_{\mathbf{2}}\right)\right\rangle, \text { if } s_{\mathbf{1}} \neq \text { the accepting s } \\ s, \text { otherwise. }\end{array}\right.$

Let $T$ be a Turing machine structure and let $s$ be a State of $T$. The functor Computation $(s)$ yielding a function from $\mathbb{N}$ into : the control states of $T, \mathbb{Z}$, (the symbols of $T)^{\mathbb{Z}}$ : is defined by:

(Def. 8) $($ Computation $(s))(0)=s$ and for every $i$ holds $($ Computation $(s))(i+1)=$ Following $(($ Computation $(s))(i))$. 
In the sequel $T$ is a Turing machine structure and $s$ is a State of $T$. We now state several propositions:

(9) Let $T$ be a Turing machine structure and $s$ be a State of $T$. If $s_{1}=$ the accepting state of $T$, then $s=$ Following $(s)$.

(10) $($ Computation $(s))(0)=s$.

(11) $($ Computation $(s))(k+1)=\operatorname{Following}((\operatorname{Computation}(s))(k))$.

(12) $($ Computation $(s))(1)=$ Following $(s)$.

(13) $($ Computation $(s))(i+k)=($ Computation $((\operatorname{Computation}(s))(i)))(k)$.

(14) If $i \leq j$ and Following $((\operatorname{Computation}(s))(i))=(\operatorname{Computation}(s))(i)$, then $(\operatorname{Computation}(s))(j)=$ $($ Computation $(s))(i)$.

(15) If $i \leq j$ and $($ Computation $(s))(i)_{\mathbf{1}}=$ the accepting state of $T$, then $(\operatorname{Computation}(s))(j)=$ $($ Computation $(s))(i)$.

Let $T$ be a Turing machine structure and let $s$ be a State of $T$. We say that $s$ is accepting if and only if:

(Def. 9) There exists $k$ such that $($ Computation $(s))(k)_{1}=$ the accepting state of $T$.

Let $T$ be a Turing machine structure and let $s$ be a State of $T$. Let us assume that $s$ is accepting. The functor Result $(s)$ yields a State of $T$ and is defined as follows:

(Def. 10) There exists $k$ such that $\operatorname{Result}(s)=(\operatorname{Computation}(s))(k)$ and $(\operatorname{Computation}(s))(k)_{\mathbf{1}}=$ the accepting state of $T$.

One can prove the following proposition

(16) Let $T$ be a Turing machine structure and $s$ be a State of $T$. Suppose $s$ is accepting. Then there exists a natural number $k$ such that

(i) $($ Computation $(s))(k)_{1}=$ the accepting state of $T$,

(ii) $\operatorname{Result}(s)=($ Computation $(s))(k)$, and

(iii) for every natural number $i$ such that $i<k$ holds $($ Computation $(s))(i)_{\mathbf{1}} \neq$ the accepting state of $T$.

Let $A, B$ be non empty sets and let $y$ be a set. Let us assume that $y \in B$. The functor $\operatorname{id}(A, B, y)$ yields a function from $A$ into $[: A, B:]$ and is defined by:

(Def. 11) For every element $x$ of $A$ holds $(\operatorname{id}(A, B, y))(x)=\langle x, y\rangle$.

The function SumTran from $\left[: \operatorname{Seg}_{M} 5,\{0,1\}:\right]$ into $\left[: \operatorname{Seg}_{M} 5,\{0,1\},\{-1,0,1\}:\right]$ is defined by:

(Def. 12) SumTran $=\operatorname{id}\left(\left[: \operatorname{Seg}_{M} 5,\{0,1\}:\right],\{-1,0,1\}, 0\right)+\cdot(\langle 0,0\rangle \longmapsto\langle 0,0,1\rangle)+\cdot(\langle 0,1\rangle \longmapsto\langle 1,0$, $1\rangle)+\cdot(\langle 1,1\rangle \longmapsto\langle 1,1,1\rangle)+\cdot(\langle 1,0\rangle \longmapsto\langle 2,1,1\rangle)+\cdot(\langle 2,1\rangle \longmapsto\langle 2,1,1\rangle)+\cdot(\langle 2,0\rangle \longmapsto\langle 3,0,-1\rangle)+\cdot(\langle 3$, $1\rangle \longmapsto\langle 4,0,-1\rangle)+\cdot(\langle 4,1\rangle \stackrel{\bullet}{\longmapsto}\langle 4,1,-1\rangle)+\cdot(\langle 4,0\rangle \vdash\langle 5,0,0\rangle)$.

Next we state the proposition

(17) $\operatorname{SumTran}(\langle 0,0\rangle)=\langle 0,0,1\rangle$ and $\operatorname{SumTran}(\langle 0,1\rangle)=\langle 1,0,1\rangle$ and $\operatorname{SumTran}(\langle 1,1\rangle)=\langle 1$, $1,1\rangle$ and $\operatorname{SumTran}(\langle 1,0\rangle)=\langle 2,1,1\rangle$ and $\operatorname{SumTran}(\langle 2,1\rangle)=\langle 2,1,1\rangle$ and $\operatorname{SumTran}(\langle 2$, $0\rangle)=\langle 3,0,-1\rangle$ and $\operatorname{SumTran}(\langle 3,1\rangle)=\langle 4,0,-1\rangle$ and $\operatorname{SumTran}(\langle 4,1\rangle)=\langle 4,1,-1\rangle$ and $\operatorname{SumTran}(\langle 4,0\rangle)=\langle 5,0,0\rangle$.

Let $T$ be a Turing machine structure, let $t$ be a tape of $T$, and let $i, j$ be integers. We say that $t$ is 1 between $i, j$ if and only if:

(Def. 13) $t(i)=0$ and $t(j)=0$ and for every integer $k$ such that $i<k$ and $k<j$ holds $t(k)=1$. 
Let $f$ be a finite sequence of elements of $\mathbb{N}$, let $T$ be a Turing machine structure, and let $t$ be a tape of $T$. We say that $t$ stores data $f$ if and only if:

(Def. 14) For every natural number $i$ such that $1 \leq i$ and $i<\operatorname{len} f$ holds $t$ is 1 between $\sum \operatorname{Prefix}(f, i)+$ $2 \cdot(i-1), \sum \operatorname{Prefix}(f, i+1)+2 \cdot i$.

Next we state several propositions:

(18) Let $T$ be a Turing machine structure, $t$ be a tape of $T$, and $s, n$ be natural numbers. If $t$ stores data $\langle s, n\rangle$, then $t$ is 1 between $s, s+n+2$.

(19) Let $T$ be a Turing machine structure, $t$ be a tape of $T$, and $s, n$ be natural numbers. If $t$ is 1 between $s, s+n+2$, then $t$ stores data $\langle s, n\rangle$.

(20) Let $T$ be a Turing machine structure, $t$ be a tape of $T$, and $s, n$ be natural numbers. Suppose $t$ stores data $\langle s, n\rangle$. Then $t(s)=0$ and $t(s+n+2)=0$ and for every integer $i$ such that $s<i$ and $i<s+n+2$ holds $t(i)=1$.

(21) Let $T$ be a Turing machine structure, $t$ be a tape of $T$, and $s, n_{1}, n_{2}$ be natural numbers. Suppose $t$ stores data $\left\langle s, n_{1}, n_{2}\right\rangle$. Then $t$ is 1 between $s, s+n_{1}+2$ and 1 between $s+n_{1}+2$, $s+n_{1}+n_{2}+4$.

(22) Let $T$ be a Turing machine structure, $t$ be a tape of $T$, and $s, n_{1}, n_{2}$ be natural numbers. Suppose $t$ stores data $\left\langle s, n_{1}, n_{2}\right\rangle$. Then

(i) $t(s)=0$,

(ii) $t\left(s+n_{1}+2\right)=0$,

(iii) $t\left(s+n_{1}+n_{2}+4\right)=0$,

(iv) for every integer $i$ such that $s<i$ and $i<s+n_{1}+2$ holds $t(i)=1$, and

(v) for every integer $i$ such that $s+n_{1}+2<i$ and $i<s+n_{1}+n_{2}+4$ holds $t(i)=1$.

(23) Let $f$ be a finite sequence of elements of $\mathbb{N}$ and $s$ be a natural number. If len $f \geq 1$, then $\sum \operatorname{Prefix}(\langle s\rangle \frown f, 1)=s$ and $\sum \operatorname{Prefix}(\langle s\rangle \frown f, 2)=s+f_{1}$.

(24) Let $f$ be a finite sequence of elements of $\mathbb{N}$ and $s$ be a natural number. Suppose len $f \geq 3$. Then $\sum \operatorname{Prefix}(\langle s\rangle \frown f, 1)=s$ and $\sum \operatorname{Prefix}(\langle s\rangle \frown f, 2)=s+f_{1}$ and $\sum \operatorname{Prefix}(\langle s\rangle \frown f, 3)=s+$ $f_{1}+f_{2}$ and $\sum \operatorname{Prefix}(\langle s\rangle \frown f, 4)=s+f_{1}+f_{2}+f_{3}$.

(25) Let $T$ be a Turing machine structure, $t$ be a tape of $T, s$ be a natural number, and $f$ be a finite sequence of elements of $\mathbb{N}$. If len $f \geq 1$ and $t$ stores data $\langle s\rangle \frown f$, then $t$ is 1 between $s$, $s+f_{1}+2$.

(26) Let $T$ be a Turing machine structure, $t$ be a tape of $T, s$ be a natural number, and $f$ be a finite sequence of elements of $\mathbb{N}$. Suppose len $f \geq 3$ and $t$ stores data $\langle s\rangle\urcorner f$. Then $t$ is 1 between $s, s+f_{1}+2,1$ between $s+f_{1}+2, s+f_{1}+f_{2}+4$, and 1 between $s+f_{1}+f_{2}+4$, $s+f_{1}+f_{2}+f_{3}+6$.

\section{Summation of Two Natural Numbers}

The strict Turing machine structure SumTuring is defined by the conditions (Def. 15).

(Def. 15)(i) The symbols of SumTuring $=\{0,1\}$,

(ii) the control states of SumTuring $=\operatorname{Seg}_{M} 5$,

(iii) the transition of SumTuring = SumTran,

(iv) the initial state of SumTuring $=0$, and

(v) the accepting state of SumTuring $=5$.

One can prove the following propositions: 
(27) Let $T$ be a Turing machine structure, $s$ be a State of $T$, and $p, h, t$ be sets. If $s=\langle p, h, t\rangle$, then $\operatorname{Head}(s)=h$.

(28) Let $T$ be a Turing machine structure, $t$ be a tape of $T, h$ be an integer, and $s$ be a symbol of $T$. If $t(h)=s$, then Tape-Chg $(t, h, s)=t$.

(29) Let $T$ be a Turing machine structure, $s$ be a State of $T$, and $p, h, t$ be sets. Suppose $s=\langle p, h, t\rangle$ and $p \neq$ the accepting state of $T$. Then Following $(s)=\left\langle s\right.$-target $_{1}, \operatorname{Head}(s)+$ $\operatorname{offset}(s$-target $)$, Tape-Chg $\left(s_{\mathbf{3}}, \operatorname{Head}(s), s\right.$-target 2$\left.)\right\rangle$.

(30) Let $T$ be a Turing machine structure, $t$ be a tape of $T, h$ be an integer, $s$ be a symbol of $T$, and $i$ be a set. Then $(\operatorname{Tape}-\operatorname{Chg}(t, h, s))(h)=s$ and if $i \neq h$, then $(\operatorname{Tape}-\operatorname{Chg}(t, h, s))(i)=t(i)$.

(31) Let $s$ be a State of SumTuring, $t$ be a tape of SumTuring, and $h_{1}, n_{1}, n_{2}$ be natural numbers. Suppose $s=\left\langle 0, h_{1}, t\right\rangle$ and $t$ stores data $\left\langle h_{1}, n_{1}, n_{2}\right\rangle$. Then $s$ is accepting and $(\operatorname{Result}(s))_{2}=$ $1+h_{1}$ and $(\operatorname{Result}(s))_{3}$ stores data $\left\langle 1+h_{1}, n_{1}+n_{2}\right\rangle$.

Let $T$ be a Turing machine structure and let $F$ be a function. We say that $T$ computes $F$ if and only if the condition (Def. 16) is satisfied.

(Def. 16) Let $s$ be a State of $T, t$ be a tape of $T, a$ be a natural number, and $x$ be a finite sequence of elements of $\mathbb{N}$. Suppose $x \in \operatorname{dom} F$ and $s=\langle$ the initial state of $T, a, t\rangle$ and $t$ stores data $\langle a\rangle^{\frown} x$. Then $s$ is accepting and there exist natural numbers $b, y$ such that $(\operatorname{Result}(s))_{2}=b$ and $y=F(x)$ and $(\operatorname{Result}(s))_{3}$ stores data $\langle b\rangle \frown\langle y\rangle$.

We now state two propositions:

(32) $\operatorname{dom}[+] \subseteq \mathbb{N}^{2}$.

(33) SumTuring computes $[+]$

\section{Computing Successor Function}

The function SuccTran from $\left[: \operatorname{Seg}_{M} 4,\{0,1\}:\right]$ into $\left[: \operatorname{Seg}_{M} 4,\{0,1\},\{-1,0,1\}:\right]$ is defined by:

(Def. 17) SuccTran $=\operatorname{id}\left(\left[: \operatorname{Seg}_{M} 4,\{0,1\}:\right],\{-1,0,1\}, 0\right)+\cdot(\langle 0,0\rangle \mapsto\langle 1,0,1\rangle)+\cdot(\langle 1,1\rangle \longmapsto\langle 1,1$, $1\rangle)+\cdot(\langle 1,0\rangle \longmapsto\langle 2,1,1\rangle)+\cdot(\langle 2,0\rangle \longmapsto\langle 3,0,-1\rangle)+\cdot(\langle 2,1\rangle \longmapsto\langle 3,0,-1\rangle)+\cdot(\langle 3,1\rangle \longmapsto\langle 3,1$, $-1\rangle)+\cdot(\langle 3,0\rangle \mapsto\langle 4,0,0\rangle)$.

We now state the proposition

(34) $\operatorname{SuccTran}(\langle 0,0\rangle)=\langle 1,0,1\rangle$ and $\operatorname{SuccTran}(\langle 1,1\rangle)=\langle 1,1,1\rangle$ and $\operatorname{SuccTran}(\langle 1,0\rangle)=$ $\langle 2,1,1\rangle$ and $\operatorname{SuccTran}(\langle 2,0\rangle)=\langle 3,0,-1\rangle$ and $\operatorname{SuccTran}(\langle 2,1\rangle)=\langle 3,0,-1\rangle$ and $\operatorname{SuccTran}(\langle 3,1\rangle)=\langle 3,1,-1\rangle$ and $\operatorname{SuccTran}(\langle 3,0\rangle)=\langle 4,0,0\rangle$.

The strict Turing machine structure SuccTuring is defined by the conditions (Def. 18).

(Def. 18)(i) The symbols of SuccTuring $=\{0,1\}$,

(ii) the control states of SuccTuring $=\operatorname{Seg}_{M} 4$,

(iii) the transition of SuccTuring = SuccTran,

(iv) the initial state of SuccTuring $=0$, and

(v) the accepting state of SuccTuring $=4$.

The following two propositions are true:

(36) Let $s$ be a State of SuccTuring, $t$ be a tape of SuccTuring, and $h_{1}, n$ be natural numbers. Suppose $s=\left\langle 0, h_{1}, t\right\rangle$ and $t$ stores data $\left\langle h_{1}, n\right\rangle$. Then $s$ is accepting and $(\operatorname{Result}(s))_{2}=h_{1}$ and $(\operatorname{Result}(s))_{3}$ stores data $\left\langle h_{1}, n+1\right\rangle$.

(37) SuccTuring computes $\operatorname{succ}_{1}(1)$.

\footnotetext{
1 The proposition (35) has been removed.
} 


\section{COMPuting Zero Function}

The function ZeroTran from [: $\operatorname{Seg}_{M} 4,\{0,1\}$ :] into [: $\left.\operatorname{Seg}_{M} 4,\{0,1\},\{-1,0,1\}:\right]$ is defined by:

(Def. 19) ZeroTran $=\operatorname{id}\left(\left[: \operatorname{Seg}_{M} 4,\{0,1\}:\right],\{-1,0,1\}, 1\right)+\cdot(\langle 0,0\rangle \mapsto\langle 1,0,1\rangle)+\cdot(\langle 1,1\rangle \vdash\langle 2,1$, $1\rangle)+\cdot(\langle 2,0\rangle \longmapsto\langle 3,0,-1\rangle)+\cdot(\langle 2,1\rangle \longmapsto\langle 3,0,-1\rangle)+\cdot(\langle 3,1\rangle \longmapsto\langle 4,1,-1\rangle)$.

The following proposition is true

(38) ZeroTran $(\langle 0,0\rangle)=\langle 1,0,1\rangle$ and ZeroTran $(\langle 1,1\rangle)=\langle 2,1,1\rangle$ and ZeroTran $(\langle 2,0\rangle)=\langle 3$, $0,-1\rangle$ and ZeroTran $(\langle 2,1\rangle)=\langle 3,0,-1\rangle$ and ZeroTran $(\langle 3,1\rangle)=\langle 4,1,-1\rangle$.

The strict Turing machine structure ZeroTuring is defined by the conditions (Def. 20).

(Def. 20)(i) The symbols of ZeroTuring $=\{0,1\}$,

(ii) the control states of ZeroTuring $=\operatorname{Seg}_{M} 4$,

(iii) the transition of ZeroTuring $=$ ZeroTran,

(iv) the initial state of ZeroTuring $=0$, and

(v) the accepting state of ZeroTuring $=4$.

Next we state two propositions:

(39) Let $s$ be a State of ZeroTuring, $t$ be a tape of ZeroTuring, $h_{1}$ be a natural number, and $f$ be a finite sequence of elements of $\mathbb{N}$. Suppose len $f \geq 1$ and $s=\left\langle 0, h_{1}, t\right\rangle$ and $t$ stores data $\left\langle h_{1}\right\rangle^{\frown} f$. Then $s$ is accepting and $(\operatorname{Result}(s))_{2}=h_{1}$ and $(\operatorname{Result}(s))_{3}$ stores data $\left\langle h_{1}, 0\right\rangle$.

(40) If $n \geq 1$, then ZeroTuring computes $\operatorname{const}_{n}(0)$.

\section{COMPUTING $n$-ARY PROJECT FUNCTION}

The function $n$-proj3Tran from $\left[: \operatorname{Seg}_{M} 3,\{0,1\}:\right]$ into $\left[: \operatorname{Seg}_{M} 3,\{0,1\},\{-1,0,1\}:\right]$ is defined as follows:

(Def. 21) $n$-proj3Tran $=\operatorname{id}\left(\left[: \operatorname{Seg}_{M} 3,\{0,1\}:\right],\{-1,0,1\}, 0\right)+\cdot(\langle 0,0\rangle \vdash \cdot\langle 1,0,1\rangle)+\cdot(\langle 1,1\rangle \vdash\langle 1$, $0,1\rangle)+\cdot(\langle 1,0\rangle \longmapsto\langle 2,0,1\rangle)+\cdot(\langle 2,1\rangle \longmapsto \cdot\langle 2,0,1\rangle)+\cdot(\langle 2,0\rangle \longmapsto\langle 3,0,0\rangle)$.

The following proposition is true

(41) $n$-proj3Tran $(\langle 0,0\rangle)=\langle 1,0,1\rangle$ and $n-\operatorname{proj} 3 \operatorname{Tran}(\langle 1,1\rangle)=\langle 1,0,1\rangle$ and $n-\operatorname{proj} 3 \operatorname{Tran}(\langle 1$, $0\rangle)=\langle 2,0,1\rangle$ and $n-\operatorname{proj} 3 \operatorname{Tran}(\langle 2,1\rangle)=\langle 2,0,1\rangle$ and $n-\operatorname{proj} 3 \operatorname{Tran}(\langle 2,0\rangle)=\langle 3,0,0\rangle$.

The strict Turing machine structure $n$-proj3Turing is defined by the conditions (Def. 22).

(Def. 22)(i) The symbols of $n$-proj3Turing $=\{0,1\}$,

(ii) the control states of $n$-proj3Turing $=\operatorname{Seg}_{M} 3$,

(iii) the transition of $n$-proj3Turing $=n$-proj3Tran,

(iv) the initial state of $n$-proj3Turing $=0$, and

(v) the accepting state of $n$-proj3Turing $=3$.

One can prove the following propositions:

(42) Let $s$ be a State of $n$-proj3Turing, $t$ be a tape of $n$-proj3Turing, $h_{1}$ be a natural number, and $f$ be a finite sequence of elements of $\mathbb{N}$. Suppose len $f \geq 3$ and $s=\left\langle 0, h_{1}, t\right\rangle$ and $t$ stores data $\left\langle h_{1}\right\rangle^{\frown} f$. Then $s$ is accepting and $(\operatorname{Result}(s))_{2}=h_{1}+f_{1}+f_{2}+4$ and $(\operatorname{Result}(s))_{3}$ stores data $\left\langle h_{1}+f_{1}+f_{2}+4, f_{3}\right\rangle$.

(43) If $n \geq 3$, then $n$-proj3Turing computes $\operatorname{proj}_{n}(3)$. 


\section{Combining Two Turing Machines into One}

Let $t_{1}, t_{2}$ be Turing machine structures. The functor SeqStates $\left(t_{1}, t_{2}\right)$ yields a finite non empty set and is defined by the condition (Def. 23).

(Def. 23) SeqStates $\left(t_{1}, t_{2}\right)=\left[\right.$ : the control states of $t_{1},\left\{\right.$ the initial state of $\left.\left.t_{2}\right\}:\right] \cup[:\{$ the accepting state of $\left.t_{1}\right\}$, the control states of $t_{2}:$.

We now state four propositions:

(44) Let $t_{1}, t_{2}$ be Turing machine structures. Then

(i) $\left\langle\right.$ the initial state of $t_{1}$, the initial state of $\left.t_{2}\right\rangle \in \operatorname{SeqStates}\left(t_{1}, t_{2}\right)$, and

(ii) $\left\langle\right.$ the accepting state of $t_{1}$, the accepting state of $\left.t_{2}\right\rangle \in \operatorname{SeqStates}\left(t_{1}, t_{2}\right)$.

(45) For all Turing machine structures $s, t$ and for every state $x$ of $s$ holds $\langle x$, the initial state of $t\rangle \in \operatorname{SeqStates}(s, t)$.

(46) For all Turing machine structures $s, t$ and for every state $x$ of $t$ holds $\langle$ the accepting state of $s, x\rangle \in \operatorname{SeqStates}(s, t)$.

(47) Let $s, t$ be Turing machine structures and $x$ be an element of SeqStates $(s, t)$. Then there exists a state $x_{1}$ of $s$ and there exists a state $x_{2}$ of $t$ such that $x=\left\langle x_{1}, x_{2}\right\rangle$.

Let $s, t$ be Turing machine structures and let $x$ be a transition-target of $s$. The functor $1{ }^{\text {st }} \operatorname{Seq} \operatorname{Tran}(s, t, x)$ yields an element of $[: \operatorname{SeqStates}(s, t)$, (the symbols of $s) \cup($ the symbols of $t),\{-1,0,1\}:]$ and is defined by:

(Def. 24) $1^{\text {st }} \operatorname{Seq} \operatorname{Tran}(s, t, x)=\left\langle\left\langle x_{\mathbf{1}}\right.\right.$, the initial state of $\left.\left.t\right\rangle, x_{\mathbf{2}}, x_{\mathbf{3}}\right\rangle$.

Let $s, t$ be Turing machine structures and let $x$ be a transition-target of $t$. The functor $2^{\text {nd }} \operatorname{Seq} \operatorname{Tran}(s, t, x)$ yields an element of [:SeqStates $(s, t)$, (the symbols of $s) \cup($ the symbols of $t),\{-1,0,1\}:]$ and is defined as follows:

(Def. 25) $\quad 2^{\text {nd }} \operatorname{SeqTran}(s, t, x)=\left\langle\left\langle\right.\right.$ the accepting state of $\left.\left.s, x_{\mathbf{1}}\right\rangle, x_{\mathbf{2}}, x_{\mathbf{3}}\right\rangle$.

Let $s, t$ be Turing machine structures and let $x$ be an element of $\operatorname{SeqStates}(s, t)$. Then $x_{1}$ is a state of $s$. Then $x_{2}$ is a state of $t$.

Let $s, t$ be Turing machine structures and let $x$ be an element of $[: \operatorname{SeqStates}(s, t)$, (the symbols of $s) \cup($ the symbols of $t):$. The functor $1^{\text {st }}$ SeqState $x$ yields a state of $s$ and is defined as follows:

(Def. 26) $1^{\text {st }}$ SeqState $x=\left(x_{1}\right)_{1}$.

The functor $2^{\text {nd }}$ SeqState $x$ yields a state of $t$ and is defined as follows:

(Def. 27) $2^{\text {nd }}$ SeqState $x=\left(x_{1}\right)_{2}$.

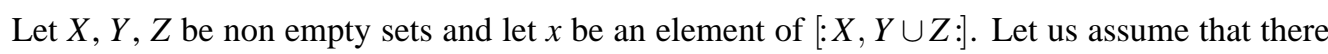
exist a set $u$ and an element $y$ of $Y$ such that $x=\langle u, y\rangle$. The functor $1^{\text {st }}$ SeqSymbol $x$ yielding an element of $Y$ is defined by:

(Def. 28) $1^{\text {st }}$ SeqSymbol $x=x_{2}$.

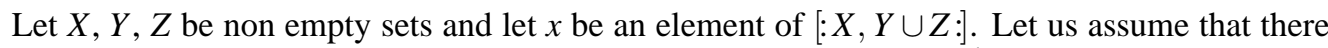
exist a set $u$ and an element $z$ of $Z$ such that $x=\langle u, z\rangle$. The functor $2^{\text {nd }}$ SeqSymbol $x$ yielding an element of $Z$ is defined as follows:

(Def. 29) $2^{\text {nd }}$ SeqSymbol $x=x_{2}$.

Let $s, t$ be Turing machine structures and let $x$ be an element of $[: \operatorname{SeqStates}(s, t)$, (the symbols of $s) \cup($ the symbols of $t):$. The functor $\operatorname{SeqTran}(s, t, x)$ yielding an element of $[: \operatorname{SeqStates}(s, t),($ the symbols of $s) \cup($ the symbols of $t),\{-1,0,1\}:]$ is defined as follows: 
(Def. 30) SeqTran $(s, t, x)=\left\{\begin{array}{l}1^{\text {st }} \operatorname{SeqT} \operatorname{Tran}\left(s, t,(\text { the transition of } s)\left(\left\langle 1^{\text {st }} \text { SeqState } x, 1^{\text {st }} \text { SeqSymbol } x\right\rangle\right)\right), \text { if thereexists a state } p \\ 2^{\text {nd }} \operatorname{SeqTran}\left(s, t,(\text { the transition of } t)\left(\left\langle 2^{\text {nd }} \operatorname{SeqState} x, 2^{\text {nd }} \operatorname{SeqSymbol} x\right\rangle\right)\right), \text { if thereexists a state } \\ \left\langle x_{\mathbf{1}}, x_{\mathbf{2}},-1\right\rangle, \text { otherwise. }\end{array}\right.$

Let $s, t$ be Turing machine structures. The functor $\operatorname{SeqTran}(s, t)$ yielding a function from $[: \operatorname{SeqStates}(s, t)$, (the symbols of $s) \cup($ the symbols of $t):]$ into [: SeqStates $(s, t)$, (the symbols of $s) \cup($ the symbols of $t),\{-1,0,1\}$ :] is defined by:

(Def. 31) For every element $x$ of [: SeqStates $(s, t)$, (the symbols of $s) \cup($ the symbols of $t)$ :] holds $(\operatorname{SeqTran}(s, t))(x)=\operatorname{SeqTran}(s, t, x)$.

Let $T_{1}, T_{2}$ be Turing machine structures. The functor $T_{1} ; T_{2}$ yielding a strict Turing machine structure is defined by the conditions (Def. 32).

(Def. 32)(i) The symbols of $T_{1} ; T_{2}=\left(\right.$ the symbols of $\left.T_{1}\right) \cup$ (the symbols of $T_{2}$ ),

(ii) the control states of $T_{1} ; T_{2}=\operatorname{SeqStates}\left(T_{1}, T_{2}\right)$,

(iii) the transition of $T_{1} ; T_{2}=\operatorname{SeqTran}\left(T_{1}, T_{2}\right)$,

(iv) the initial state of $T_{1} ; T_{2}=\left\langle\right.$ the initial state of $T_{1}$, the initial state of $\left.T_{2}\right\rangle$, and

(v) the accepting state of $T_{1} ; T_{2}=\left\langle\right.$ the accepting state of $T_{1}$, the accepting state of $\left.T_{2}\right\rangle$.

The following propositions are true:

(48) Let $T_{1}, T_{2}$ be Turing machine structures, $g$ be a transition-target of $T_{1}, p$ be a state of $T_{1}$, and $y$ be a symbol of $T_{1}$. Suppose $p \neq$ the accepting state of $T_{1}$ and $g=$ (the transition of $\left.T_{1}\right)(\langle p, y\rangle)$. Then (the transition of $\left.T_{1} ; T_{2}\right)\left(\left\langle\left\langle p\right.\right.\right.$, the initial state of $\left.\left.\left.T_{2}\right\rangle, y\right\rangle\right)=\left\langle\left\langle g_{1}\right.\right.$, the initial state of $\left.\left.T_{2}\right\rangle, g_{2}, g_{3}\right\rangle$.

(49) Let $T_{1}, T_{2}$ be Turing machine structures, $g$ be a transition-target of $T_{2}, q$ be a state of $T_{2}$, and $y$ be a symbol of $T_{2}$. Suppose $g=\left(\right.$ the transition of $\left.T_{2}\right)(\langle q, y\rangle)$. Then (the transition of $\left.T_{1} ; T_{2}\right)\left(\left\langle\left\langle\right.\right.\right.$ the accepting state of $\left.\left.\left.T_{1}, q\right\rangle, y\right\rangle\right)=\left\langle\left\langle\right.\right.$ the accepting state of $\left.\left.T_{1}, g_{\mathbf{1}}\right\rangle, g_{\mathbf{2}}, g_{\mathbf{3}}\right\rangle$.

(50) Let $T_{1}, T_{2}$ be Turing machine structures, $s_{1}$ be a State of $T_{1}, h$ be a natural number, $t$ be a tape of $T_{1}, s_{2}$ be a State of $T_{2}$, and $s_{3}$ be a State of $T_{1} ; T_{2}$. Suppose that

(i) $s_{1}$ is accepting,

(ii) $s_{1}=\left\langle\right.$ the initial state of $\left.T_{1}, h, t\right\rangle$,

(iii) $s_{2}$ is accepting,

(iv) $s_{2}=\left\langle\right.$ the initial state of $\left.T_{2},\left(\operatorname{Result}\left(s_{1}\right)\right)_{2},\left(\operatorname{Result}\left(s_{1}\right)\right)_{3}\right\rangle$, and

(v) $s_{3}=\left\langle\right.$ the initial state of $\left.T_{1} ; T_{2}, h, t\right\rangle$.

Then $s_{3}$ is accepting and $\left(\operatorname{Result}\left(s_{3}\right)\right)_{\mathbf{2}}=\left(\operatorname{Result}\left(s_{2}\right)\right)_{\mathbf{2}}$ and $\left(\operatorname{Result}\left(s_{3}\right)\right)_{\mathbf{3}}=\left(\operatorname{Result}\left(s_{2}\right)\right)_{\mathbf{3}}$.

(51) Let $t_{3}, t_{4}$ be Turing machine structures and $t$ be a tape of $t_{3}$. If the symbols of $t_{3}=$ the symbols of $t_{4}$, then $t$ is a tape of $t_{3} ; t_{4}$.

(52) Let $t_{3}, t_{4}$ be Turing machine structures and $t$ be a tape of $t_{3} ; t_{4}$. Suppose the symbols of $t_{3}=$ the symbols of $t_{4}$. Then $t$ is a tape of $t_{3}$ and a tape of $t_{4}$.

(53) Let $f$ be a finite sequence of elements of $\mathbb{N}, t_{3}, t_{4}$ be Turing machine structures, $t_{1}$ be a tape of $t_{3}$, and $t_{2}$ be a tape of $t_{4}$. If $t_{1}=t_{2}$ and $t_{1}$ stores data $f$, then $t_{2}$ stores data $f$.

(54) Let $s$ be a State of ZeroTuring; SuccTuring, $t$ be a tape of ZeroTuring, and $h_{1}, n$ be natural numbers. Suppose $s=\left\langle\langle 0,0\rangle, h_{1}, t\right\rangle$ and $t$ stores data $\left\langle h_{1}, n\right\rangle$. Then $s$ is accepting and $(\operatorname{Result}(s))_{2}=h_{1}$ and $(\operatorname{Result}(s))_{3}$ stores data $\left\langle h_{1}, 1\right\rangle$. 


\section{REFERENCES}

[1] Grzegorz Bancerek. The fundamental properties of natural numbers. Journal of Formalized Mathematics, 1, 1989. http://mizar. org/JFM/Voll/nat_1.html

[2] Grzegorz Bancerek. The ordinal numbers. Journal of Formalized Mathematics, 1, 1989. http://mizar.org/JFM/Vol1/ordinal1. html.

[3] Grzegorz Bancerek. Sequences of ordinal numbers. Journal of Formalized Mathematics, 1, 1989. http://mizar.org/JFM/Vol1/ ordinal2.html

[4] Grzegorz Bancerek and Krzysztof Hryniewiecki. Segments of natural numbers and finite sequences. Journal of Formalized Mathematics, 1, 1989. http://mizar.org/JFM/Vol1/finseq_1.html

[5] Grzegorz Bancerek and Piotr Rudnicki. The set of primitive recursive functions. Journal of Formalized Mathematics, 13, 2001. http: //mizar.org/JFM/Vol13/comput_1.html

[6] Czesław Byliński. Functions and their basic properties. Journal of Formalized Mathematics, 1, 1989. http://mizar.org/JFM/Vol1/ funct_1.html

[7] Czesław Byliński. Functions from a set to a set. Journal of Formalized Mathematics, 1, 1989. http://mizar.org/JFM/Vol1/funct_ 2.html

[8] Czesław Byliński. Partial functions. Journal of Formalized Mathematics, 1, 1989. http://mizar.org/JFM/Vol1/part fun1.html.

[9] Czesław Byliński. Some basic properties of sets. Journal of Formalized Mathematics, 1, 1989. http://mizar.org/JFM/Vol1/ zfmisc_1.html

[10] Czesław Byliński. A classical first order language. Journal of Formalized Mathematics, 2, 1990. http://mizar.org/JFM/Vol2/cqC_ lang.html

[11] Czesław Byliński. Finite sequences and tuples of elements of a non-empty sets. Journal of Formalized Mathematics, 2, 1990. http: //mizar.org/JFM/Vol2/finseq_2.html

[12] Czesław Byliński. The modification of a function by a function and the iteration of the composition of a function. Journal of Formalized Mathematics, 2, 1990. http://mizar.org/JFM/Vol2/funct_4.html

[13] Jing-Chao Chen. A small computer model with push-down stack. Journal of Formalized Mathematics, 11, 1999. http://mizar.org/ JFM/Vol11/scmpds_1.html

[14] Agata Darmochwał. Finite sets. Journal of Formalized Mathematics, 1, 1989. http://mizar.org/JFM/Vol1/finset_1.html

[15] Krzysztof Hryniewiecki. Basic properties of real numbers. Journal of Formalized Mathematics, 1, 1989. http://mizar.org/JFM/ Vol1/real_1.html

[16] Dariusz Surowik. Cyclic groups and some of their properties — part I. Journal of Formalized Mathematics, 3, 1991. http://mizar. org/JFM/Vol3/gr_Cy_1.html

[17] Andrzej Trybulec. Domains and their Cartesian products. Journal of Formalized Mathematics, 1, 1989. http://mizar.org/JFM/ Voll/domain_1.html.

[18] Andrzej Trybulec. Tarski Grothendieck set theory. Journal of Formalized Mathematics, Axiomatics, 1989. http://mizar.org/JFM/ Axiomatics/tarski.html

[19] Andrzej Trybulec. Tuples, projections and Cartesian products. Journal of Formalized Mathematics, 1, 1989. http://mizar.org/JFM/ Vol1/mcart_1.html

[20] Andrzej Trybulec. Function domains and Frænkel operator. Journal of Formalized Mathematics, 2, 1990. http://mizar.org/JFM/ Vol2/fraenkel.html.

[21] Andrzej Trybulec. Subsets of real numbers. Journal of Formalized Mathematics, Addenda, 2003. http://mizar.org/JFM/Addenda/ numbers.html

[22] Michał J. Trybulec. Integers. Journal of Formalized Mathematics, 2, 1990. http://mizar.org/JFM/Vol2/int_1.html.

[23] Wojciech A. Trybulec. Pigeon hole principle. Journal of Formalized Mathematics, 2, 1990. http://mizar.org/JFM/Vol2/finseq_ 4.html

[24] Zinaida Trybulec. Properties of subsets. Journal of Formalized Mathematics, 1, 1989. http://mizar.org/JFM/Vol1/subset_1.htm1

[25] Edmund Woronowicz. Relations and their basic properties. Journal of Formalized Mathematics, 1, 1989. http://mizar.org/JFM/ Vol1/relat_1.html

Received July 27, 2001

Published January 2, 2004 